\title{
Caracterización de los emprendedores y su percepción sobre el turismo rural: Curarrehue, Chile
}

\author{
Alan Garin* \\ Universidad de La Frontera (Chile) \\ Bastian Quinteros** \\ Universidad de La Frontera (Chile)
}

\begin{abstract}
Resumen: Con la crisis del sector agrícola, ha sido necesario desarrollar nuevas actividades productivas. En consecuencia, en Curarrehue, región de La Araucanía, Chile, el turismo rural surge como un promisorio eje de desarrollo. Esta investigación caracteriza el perfil de los emprendedores turísticos y su percepción sobre la actividad. Se usó un enfoque basado en el constructivismo social, técnicas cualitativas fueron aplicadas como la entrevista semiestructurada y la metodología FODA. Los resultados muestran que los participantes son principalmente mujeres y adultos con bajo nivel de educación, con una positiva percepción de la actividad turística la cual les ha permitido mejorar sus condiciones de vida y como debilidad, los bajos niveles de asociatividad. Sus principales amenazas son las actividades relacionadas con el capitalismo global, enfrentado una mayor competencia por los recursos naturales y paisajísticos.
\end{abstract}

Palabras Clave: Turismo rural; Emprendedores; Características; Percepción; Curarrehue; Chile.

\section{Characterization of entrepreneurs and their perception of rural tourism: Curarrehue, Chile}

Abstract: With the agricultural crisis in rural sectors it has become necessary to develop new productive activities. Accordingly, in Curarrehue, in the Region of the Araucanía, Chile, rural tourism has appeared as a promising axis of development. This research characterises the profile of tourism entrepreneurs and their perceptions of tourism activity. Using a social constructivist approach, qualitative techniques were applied, e.g semi-structured interviews and SWOT analysis. The results show that participants are predominantly women and seniors with scarce formal education and a positive perception of tourism, which had led them to improve their living conditions, with low levels of partnerships found as a weakness. The main threat is posed by foreign activities related with the global capitalism that result in increased competition for natural and landscape resources.

Keywords: Rural tourism; Entrepreneurs; Characteristics; Perceptions; Curarrehue; Chile.

\section{Introduccion}

El turismo, en las últimas décadas, ha ido adquiriendo un rol significativo en las nuevas dinámicas territoriales de los espacios rurales, permitiendo el surgimiento y desarrollo de diversas iniciativas que buscan mejorar la calidad de vida de la población local (De Souza, 2012; Cardona y Sierra, 2013; Sánchez, 2014). Lo anterior se debe a la presencia de diversos factores que se han desencadenado por la globalización. Uno de estos es la pérdida de importancia de las actividades agrícolas tradicionales, lo cual ha dado lugar a un cambio relevante en la forma de interpretar y aplicar el desarrollo rural. Diversos autores como Sharpley (2002); Briedenhann y Wickens, (2004), Fuller, (2011); Flores y Barroso (2012), Komppula, 2014; Randelli, Romei y Tortora (2014) destacan que, tanto la agricultura como la

\footnotetext{
* Universidad de La Frontera (Chile); E-mail: alan.garin@ufrontera.cl

* Universidad de La Frontera (Chile); E-mail: b.quinteros01@ufromail.cl
} 
ganadería ya no son las únicas actividades productivas en el mundo rural, donde el turismo comienza a cumplir un rol destacado como una nueva fuente de ingresos. El turismo pasa a formar parte de lo que se conoce como "Nueva Ruralidad", proceso que explica las profundas transformaciones de los espacios rurales, no tan solo en términos económicos, sino que también sociales, culturales, revalorizando el paisaje natural. Lo anterior, ha permitido que la actividad se constituya en un instrumento de desarrollo para los territorios empobrecidos, por lo que se han diseñado estrategias alternativas para la regeneración económica y social, estrategias que han significado un catalizador para estimular el crecimiento económico y mejorar el nivel de vida de las comunidades locales. Como un ejemplo se puede destacar el caso de los países de África Subsahariana, donde se percibe al turismo como una de las pocas opciones viables para el desarrollo (Briedenhann y Wickens, 2004; Millán, López-Guzmán y Agudo, 2006).

Cohen (2005) menciona que surge un nuevo tipo de turista, el "post-turista". Este nuevo turista viaja en busca de experiencias distintivas y únicas. También es expresión de la diversificación y creciente sofisticación en los intereses de los viajeros que cada vez son más exigentes con respecto a la calidad de la experiencia como también el deseo de visitar lugares turísticos que ostentan una variedad de significados culturales, arqueológicos e históricos, donde el turista busca el contacto con la naturaleza, la cultura, la vida campesina y tranquilidad (Fuller, 2011; Flores y Barroso, 2011; Marujo y Cravidao, 2012; Cardona y Sierra, 2013).

Aunque son diversas las ventajas que ofrece la actividad turística en los espacios rurales, también puede generar efectos adversos para las comunidades locales y su patrimonio natural y cultural. Diversos autores han identificado que de no existir una adecuada estrategia en la gestión turística, se puede generar una saturación de los alojamientos que conlleven a una masificación del destino y que replique las pautas de comportamiento del turismo de sol y playa. Actualmente, se observa el uso de lagos y embalses con artefactos a motor, senderismo no controlado y, en algunas oportunidades, masivo. Esta situación, además, provoca una sobre explotación de los recursos que incide en la capacidad de carga y por lo tanto, la degradación de estos. (García, 1996; Costa y Barretto, 2015).

La implementación del turismo, además de los efectos ya mencionados, ha significado que en algunos territorios los campesinos abandonen sus tierras a través de mecanismos de mercado, sus costumbres y tradiciones se utilizan para fines comerciales, tercerización de la actividad rural y por consiguiente, el abandono de las actividades agrícolas tradicionales, se reproducen modelos de desarrollo que no consideran tanto las características como necesidades locales, el predominio de microempresas familiares donde el turismo es solo una actividad complementaria y que puede afectar la viabilidad económica del emprendimiento, lo que a su vez, limita el tiempo disponible para la gestión y asociatividad (Vásquez y Martin, 2011; Delgado, 2014; Milano y Gascón, 2017).

En el caso de Chile y en particular la región de La Araucanía, Chile, el turismo rural (TR), está siendo considerado como una actividad significativa para mejorar la calidad de vida de los emprendedores. No obstante lo anterior, los estudios sobre las condiciones que tiene esta actividad son escasos, especialmente aquellos que toman en consideración las características, experiencias y percepción en el contexto de su realidad social.

Considerando lo señalado, este estudio busca contribuir al conocimiento sobre el TR y que sea un aporte tanto a futuras investigaciones como a la política pública en la comuna de Curarrehue, espacio periférico de la región de La Araucanía, territorio que cuenta con un marco cultural e histórico que la distingue de otros espacios rurales y un variado paisaje donde se mezcla vegetación, fauna e importantes recursos hídricos, por lo que el objetivo fue conocer las características y percepción de los emprendedores turísticos sobre las oportunidades que les brinda realizar la actividad, sus relaciones con el municipio local, identificar los principales desafíos y amenazas que deben superar para consolidar y fortalecer su emprendimiento como una alternativa para mejorar sus condiciones vida. A la vez, se buscó identificar los diferentes recursos naturales que permitan sustentar la actividad.

En consideración a los objetivos indicados y de acuerdo a González (2011: 85) "el turismo como hoy lo conocemos es un fenómeno social, que se identifica con el desempeño de dos roles fundamentales, el de anfitrión y el de turista. Ambos roles son construcciones sociales, en el sentido de que incluyen actividades, actitudes, prácticas, significados, representaciones, códigos y pautas de conducta que los identifican y que son idealmente compartidos por muy diversos individuos".

$\mathrm{Al}$ considerar al turismo como resultado de una construcción social, la investigación se abordó a través de una mirada constructivista, desde la perspectiva del emprendedor, el cual, producto de su historia, cultura y saberes, va construyendo socialmente el espacio turístico. En este sentido, Berger y Luckmann (2006) tratan de demostrar que toda la realidad social no es otra cosa que una construcción de la misma sociedad, en las cuales los individuos y los grupos participan en la creación de esa percepción 
social, es decir, la realidad se construye socialmente. El hombre percibe la realidad y su entorno de un mundo que para él es real. Es decir, tal como lo plantean González y Palafox (2014), la investigación del turismo debe partir desde lo específico, de la realidad social, lo cual permite la comprensión y construcción de conocimiento.

Este artículo se desarrolla presentando el marco teórico, donde se hace una revisión de lo que es la "Nueva Ruralidad" y el concepto, importancia y limitaciones del turismo rural. Posteriormente se presenta el marco metodológico. A continuación los resultados y se finaliza con conclusiones.

\section{Marco teorico: significado y enfoques}

\subsection{La Nueva Ruralidad}

El concepto teórico de la Nueva ruralidad (NR) en América Latina comienza a gestarse en la década de los noventa del siglo pasado en el contexto de la profundización de la crisis del sector agrícola y del proceso de integración por medio de diversos tratados internacionales, donde la agricultura ha sido parte de la apertura de los mercados y liberalización del comercio exterior. Otro factor que refuerza la Nueva Ruralidad, corresponde a las nuevas demandas que se originan en los espacios urbanos, como por ejemplo, el surgimiento del turismo rural en contraste con el tradicional de masas (Grajales y Concheiro, 2009; Nogar, 2007))

Este nuevo enfoque sobre las dinámicas que se están desarrollando en los espacios rurales se alimenta de factores y procesos que no se fundamenta en la agricultura, ya sea social y productivamente. Tampoco considera en particular otras actividades tradicionales como la ganadería y la silvícola. El territorio es un agente principal, por lo tanto, multidimensional, articulado e integrado. Supera la visión dicotómica entre lo urbano y rural, lo que implica una nueva relación entre estos espacios, revalorizando y reconociendo lo rural como un factor de desarrollo para su población. En este sentido, la territorialidad se constituye en una expresión espacial de primer orden (Nogar, 2007; Grajales y Concheiro, 2009). Esta pluriactividad genera una estructura ocupacional cada vez más diversificada, donde el empleo rural no agrícola ocupa mayores espacios en la estructura de la población económicamente activa (Nogar, 2007; Garín, Albers y Ortega, 2011).

La transformación productiva busca articular competitiva y sustentablemente la economía del territorio hacia mercados dinámicos. Los espacios rurales vislumbran una serie de nuevos productos y servicios que se convierten en alternativa productiva y de desarrollo, como la agroindustria, turismo rural, artesanía, gastronomía, entre otras. Socialmente, se asiste a una mayor presencia de las mujeres en las actividades productivas, ya sea como mano de obra temporera, realizando emprendimientos turísticos, artesanales y gastronómicos. Surge lo que podríamos llamar el obrero o asalariado campesino (Grajales y Concheiro, 2009; Garín, 2015; Pérez, 2010). Se puede señalar, tal como lo indican Monterroso y Zizumbo (2009: 137) que en "la nueva ruralidad hay dos ideas de importancia fundamental. Por un lado está la relativa al manejo de los recursos naturales y culturales; por otro, la relacionada con la centralidad de los actores, a los que supone el centro de todo proyecto de desarrollo rural sostenible. Con base en ellas se sostiene que para que la población rural pueda hacerle frente a la pobreza debe buscar alternativas que le ayuden a solucionar problemas, lo cual lograrán sólo si van más allá de las actividades agrícolas tradicionales". Por su parte, Varisco (2016) menciona que la NR ha generado una transformación de las relaciones espaciales y sociales como también en cómo se entiende lo rural, ya no es algo dicotómico a lo urbano.

No obstante lo mencionado precedentemente, el concepto de NR ha sido cuestionado en diferentes aspectos. En este sentido Gómez (2002), sin desconocer que en el mundo rural han ocurrido trasformaciones, la N.R. ya tiene varias décadas de existencia. Lo que se observa como nuevo, es una realidad que antes se ignoraba. También se sostiene que existe un escaso desarrollo teórico, con diferentes enfoques conceptuales que no tienen una explicación consistente y donde no existe una teoría que permita una categorización más completa, sin una perspectiva histórica que dé cuenta de su origen y sin considerar los efectos de la globalización y el neoliberalismo (Llambi y Pérez, 2007; Ramírez, C., 2014; Sánchez, 2016). Siguiendo similar argumentación, Kay (2009:610) señala "El concepto de nueva ruralidad evidentemente despertó el interés de mucha gente que se ocupa de los temas rurales y estimuló nuevas investigaciones en la transformación rural en Latinoamérica, sin embargo, el término nunca ha sido desarrollado de un modo sistemático y total. A medida que se generalizó su uso, se le añadieron nuevas características a la definición de una forma asistemática y fragmentada”. 
La NR no considera a la globalización neoliberal como una fase capitalista que ha conducido los cambios tanto en los espacios urbanos como rurales, se genera una subordinación desarticulada de la agricultura en relación a la industria y alteraciones en la sociedad rural. A esto se agrega que la N.R no toma en cuenta que los procesos de globalización y privatización revelen fallas de mercado. El promover la pluriactividad sin cambiar el contexto sociocultural, es reproducir el neoliberalismo y por la tanto explotación y despojo del campesinado (Ramírez, 2014; Kay, 2007).

Independiente de la visión que se tenga de la NR, es indiscutible que en el espacio rural se observan nuevos actores, transformaciones socioculturales y actividades económicas, siendo una de ellas, el turismo rural. El turismo rural (TR) ha ido adquiriendo una mayor relevancia para el desarrollo de las comunidades locales, siendo una de los principales alternativas de los espacios rurales, desplegando un conjunto de servicios y productos que generan ingresos no agrícolas y que, en algunos casos, es un complemento a la agricultura tradicional (García, 2005; Pérez, 2010)

\subsection{Turismo rural, significado, importancia y limitaciones}

Conceptualmente, la definición de TR es compleja, ya que no existe un criterio único ni consenso sobre su significado debido a que los investigadores han desarrollado sus propias definiciones que se han basado en sus experiencias o contextos particulares (Neumeier y Pollermann, 2014; Abvram y Balan, 2015). Lo anterior se origina porque no existe una clara delimitación de lo que es considerado rural. En algunos países es una definición demográfica y en otras se consideran espacios urbanos que tienen claramente características y funciones rurales (Barke, 2004 y Hall (s/f). Otro aspecto que ayuda a esta falta de consenso, es que el TR se caracteriza por su versatilidad y diversidad. Se confunde con el ecoturismo, turismo verde o turismo de vida silvestre, agroturismo o turismo en casa del agricultor. Esto hace que la naturaleza del TR es en muchos aspectos diferentes del turismo en general, ya que se puede restringir a categorías o diversas formas de turismo como por ejemplo, el agroturismo (Lane, 1994 y Bardon, 1990). Lo anterior queda reflejado en algunas definiciones de TR como la de Barrera (2006) quien la define como aquella actividad turística realizada en el espacio rural, compuesto por una oferta integrada de ocio dirigida a una demanda cuya motivación incluye el contacto respetuoso con el entorno natural y una interrelación con la población local. Briedenhann y Wickens (2004) lo entienden como al turismo en el medio rural a un conjunto de actividades que se desarrollan en dicho entorno, excediendo el mero alojamiento y que pueden constituirse, para la población, en ingresos complementarios a a su actividad agrícola y donde las personas viven en granjas o pequeños grupos de propiedades menores a 30 residencias. Por su parte, el Instituto Nacional Agropecuario (INDAP,2012) lo considera como aquella actividad turística desarrollada en el medio rural por los usuarios y/o potenciales usuarios de la institución y que tengan como soporte la actividad silvoagropecuaria, el campo y lugares conexos, que ofrezca al cliente el compartir y experimentar la cultura y tradiciones campesinas. En esta última definición se sustentará la orientación de la investigación, ya que los emprendedores forman parte de la red de turismo rural de la institución.

Ahora bien, es relevante intentar responder algunas de las siguientes interrogantes que nos permitan entender la importancia del turismo rural. ¿Por qué el turismo rural puede ser beneficioso para el desarrollo de las comunidades locales?, ¿hay suficiente evidencia empírica sobre los beneficios que se obtienen de esta actividad?, ¿es realmente un factor que permite un desarrollo integral de los territorios? , o ¿también es posible que la actividad genere efectos adversos en los territorios en que se desarrolla? El mercado muestra un interés creciente en el campo como un espacio de ocio que ofrece zonas para la recreación al aire libre, naturaleza y cultura (Kastenholz, Carneiro, Peixeira y Lima, 2012). Otro aspecto no menor que ha incidido en el auge del TR se relaciona con la disminución y reestructuración de la actividad agrícola, por lo que esta actividad ha tenido una buena acogida en los pequeños productores, ya que han ido perdiendo competitividad en la producción de recursos naturales, por lo que se ha convertido en un complemento a los ingresos y a la vez, facilitando el redescubrimiento de los valores que poseen los recursos naturales, los edificios históricos y el folclore tradicional rural, mejorar la comercialización de productos primarios y agregar valor, fomentar el asociativismo, incorporar diversos tamaños de establecimientos y contribuir al desarrollo local (Banco Mundial y Fao, 2003;Randelli,Romei y Tortora, 2014;(Snieška, Barkauskiené, Barkauskas, 2014; Hwnag y Lee 2015)

El desarrollo del TR también impulsa a definir las relaciones entre los principales interesados, a saber, la comunidad local, los inversionistas externos y turistas. Además, a los beneficios ya mencionados, se pueden agregar los siguientes: las características de la ruralidad se conservan con integridad, la comunidad comparte el beneficio del desarrollo del turismo rural, no sólo los inversionistas se benefician 
del negocio, los turistas disfrutan de una experiencia rural memorable con alta calidad (Zou, Huang y Ding, 2014).

Dentro de lo que es el TR, en Latinoamérica, en las últimas décadas, ha surgido un nuevo modelo que apuesta por el conocimiento y experiencia compartida con las comunidades locales, pueblos indígenas y familias campesinas y que se ha denominado "Turismo Rural Comunitario" (Cañada, 2009; Sagrado, Pons, Serrano y Vera, 2016)

El turismo de base comunitaria se fundamenta en la relacion dialectica entre la comunidad receptora y el turista, no en una sobreimposicion de la comunidad sobre el turista. Es un modelo de gestion comunitaria y una estrategia de inclusion social, potencia modos de vida radicionales, relaciones socioproductivas y politicas de base comunitaria y moviliza puestos de trabajo para la produccion artesanal. Los actores sociales se diferencian de los que se involucran con el turismo rural convencional en función de la disposición para compartir su modo de vida y su patrimonio cultural y natural, pero en especial, por mantener sus actividades económicas. Se busca generar desarrollo local por medio de la sustentabilidad de los emprendimientos que favorezcan a comunidades en situación de pobreza y que sean parte en la gestión de pequeñas iniciativas (Sampaio y Coriolano, 2009; Flores, Cunha y Christoffoli, 2016; Bianchin y Alves 2017).

El Turismo rural comunitario no se encuentra exento de dificultades para implementarlo adecuadamente. En este sentido, Tejera (2010) y Gascón (2016) mencionan que debido a que el turismo no ha sido su ocupación principal, la falta de experiencia es relevante y se traduce en dificultades para la comercialización y difusión de la actividad, lo que se suma a una falta de profesionalización de quienes llevan a cabo los proyectos. Si la experiencia recae en parte de la población, la gestión no tiene el carácter de participativo y democrático ya que se desconocerá de cómo funciona la cadena de valor o cómo se gestiona la nueva actividad. Esto puede constituirse en una actividad monopólica que beneficiará a solo parte de la población, lo cual puede constituir un elemento potencial de conflicto entre los integrantes de la comunidad.

\subsection{Metodología}

En consideración a que la investigación se efectuó desde un enfoque constructivista, donde interesa conocer las características de los emprendedores y su percepción sobre sus experiencias en torno a la actividad, fue de carácter cualitativo. La recolección de datos se realizó principalmente por medio de una entrevista semiestructurada. De acuerdo a Corbetta (2007), este tipo de entrevista concede amplia libertad tanto al entrevistado como al entrevistador, y garantiza al mismo tiempo, que se van a discutir todos los temas relevantes y recopilar toda la información necesaria.

Una primera etapa consistió en una revisión bibliográfica sobre los planes de desarrollo e información de los censos de Población y Vivienda a objeto de conocer características generales de la comuna.

Como segunda etapa, se realizó una reunión de trabajo con el encargado de turismo de la comuna a fin de identificar y localizar a los emprendedores. De esta manera, se obtuvo un listado de 32 emprendimientos, 22 mujeres y 10 varones, distribuidos en diferentes sectores del territorio.

Posteriormente, se efectuó el trabajo de campo para aplicar la entrevista semiestructurada, la cual buscó conocer las características de los emprendedores y la percepción que tienen sobre la actividad. Esta se aplicó a todas las personas identificadas en la segunda etapa. Paralelamente, se analizaron los diversos atractivos naturales, culturales e históricos que sustentan la actividad de los emprendedores a través de 'trabajo de campo, fotografías tomadas por los investigadores, complementándose con revisión bibliográfica.

Finalmente, se realizó el procesamiento de la información, utilizándose el análisis de contenido inductivo en función de los objetivos planteados. Esto significa que las entrevistas se transcribieran en forma literal y a partir de la lectura, identificar aquellas frases que respondían al objetivo de la, investigación. A partir de este análisis, se recurrió a la técnica FODA (SWOT en inglés) la que permitió identificar las fortalezas y debilidades (de carácter interno) como oportunidades y amenazas (de carácter externos).La importancia radica en que en el análisis desde esta perspectiva permite conocer que factores influyen en la sustentabilidad de la actividad turística.

\section{El contexto territorial}

La comuna de Curarrehue, localizada en la Región de La Araucanía, provincia de Cautín, es limítrofe con la Republica de Argentina. Se encuentra a unos $120 \mathrm{Km}$. al oriente de la capital regional, Temuco. 
Su población, según el Censo de Población y Vivienda del año 2017 (INE, 2017) es de 7489 habitantes, de los cuales un $50.4 \%$ correspondía a población indígena.

\section{Figura 1: Localización comuna de Curarrehue}

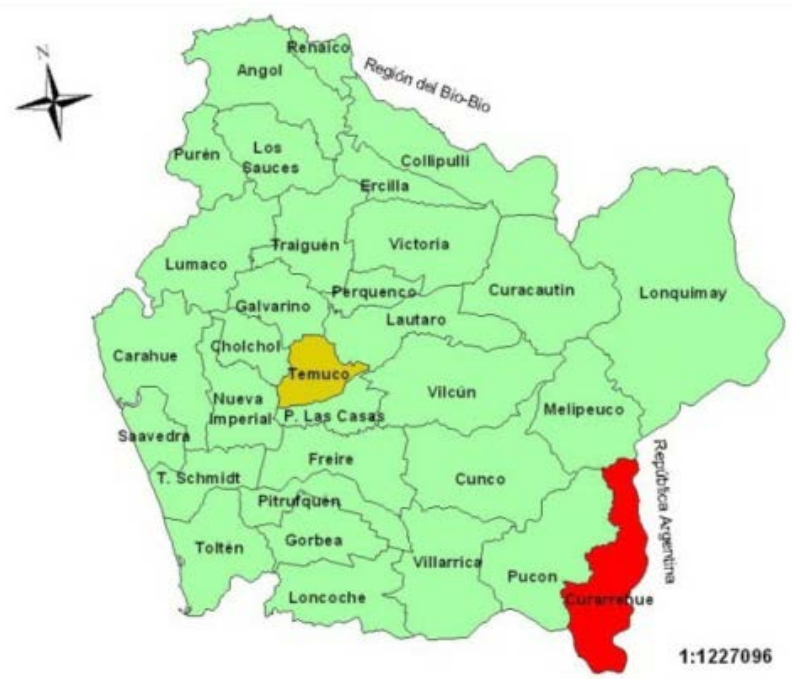

Fuente: Elaboración de los autores

El Plan de Desarrollo Comunal 2010-2016, señala que el territorio comenzó a poblarse alrededor de 1850 producto de la explotación maderera a través del río Trancura. El lugar fue conocido como el paso cordillerano de la Villa Rica siendo una ruta importante tanto para los españoles como para los pueblos originarios. Su poblamiento corresponde principalmente a la llegada de población mapuche que provenía desde el valle central de la región buscando refugio por la llegada de tropas chilenas a la región. Posteriormente, a comienzos del siglo XX, con motivo de la crisis económica que tenía el país, llegaron colonos provenientes de la zona norte y central del país. En este periodo se construye el paso internacional de Mamuil Malal que conecta con Argentina, el cual es una de las puertas de entrada a la región y al país, por lo que la comuna presenta una localización estratégica que puede ser aprovechada para su desarrollo económico, especialmente a través de la actividad turística.

Desde el punto vista productivo, su desarrollo se ha sustentado en las actividades primarias como la agricultura, ganadería y en menor medida la forestal. Fundamentalmente son actividades de subsistencia y con escaso excedente para su comercialización.

\subsection{Manifestaciones de la Nueva Ruralidad}

La NR en Curarrehue se ha manifestado por algunos cambios en la estructura de la población económicamente activa (PEA) ocupada en el sector agro-ganadero y con el surgimiento de nuevas actividades que han ido configurando una nueva dinámica socioespacial, tanto de características globales como locales. Es así que la PEA ocupada en el sector agro-ganadero, de acuerdo a los datos del Instituto Nacional de Estadísticas (INE), ha tenido importantes modificaciones entre los periodos intercensales 1992 y 2002. Es así que para el año 1992 la PEA ocupada en el sector agro-ganadero tenía una participación del 50\%, se ve reducida para el 2002 a un 30\%. A la vez, aunque la participación femenina es baja, durante el mismo periodo aumenta de un 1.2 a un 3\%. Esta tendencia debería ser confirmada en los datos censales que aún no se publican del año 2017. Otro aspecto que sobresale y que se relaciona con los empleos rurales no agrícolas, lo constituye el alza del 5 al 10\% de la población rural que se dedica a labores de construcción.

En los años noventa del siglo pasado, el espacio rural comienza a dar señales de una pérdida de la homogeneidad productiva que hasta entonces se reconocía. Por la calidad de sus recursos hídricos, este territorio se tornó atractivo para actividades no relacionadas con el mundo campesino. De esta 
manera, se instalan dos centrales de paso. La primera corresponde a la Central Hidroeléctrica de pasada Panqui, sector Huitraco, con capitales austriacos. La segunda, Añihuerraqui, cuyo propietario es la empresa GTD Negocios. La energía que se produce se entrega a un sistema interconectado que la distribuye a diferentes partes del territorio nacional, no beneficiando a los habitantes locales. Además de las centrales de paso, se localizan pisciculturas que constituyen el circuito inicial de la producción de salmón, el que finaliza en los mercados globales. Estas actividades, han generado una transformación del paisaje sociocultural, en especial las pisciculturas (Los Fiordos y Troutlodge), se observan nuevas infraestructuras productivas como camineras. A la vez, han contribuido a la emergencia de una nueva categoría laboral, el "campesino asalariado", el cual es contratado por jornadas laborales permanentes y con un salario que recibe todos los meses. De este modo, se dejan de lado las actividades asociadas a la agricultura tradicional. Desde los actores locales, se ha desarrollado el turismo rural, artesanía y gastronomía que se sustentan en el patrimonio cultural, histórico y natural que posee la comuna. Estos cambios, independiente del enfoque que se tenga de la Nueva Ruralidad, dan cuenta de una nueva configuración socioespacial, surgen nuevas actividades y nueva infraestructura, nuevos actores sociales y económicos y, además, espacios de conflictos por el uso de recursos escasos.

\subsection{Características de los emprendedores turísticos}

$\mathrm{Al}$ describir las características de los emprendedores, se observa una importante heterogeneidad en aspectos demográficos, educativos y su relación con otros emprendedores.

La NR no solo se encuentra asociada a la generación de nuevas dinámicas productivas, sino que se empieza a visibilizar la presencia de nuevos actores económicos y que, en el caso particular de la comuna y relacionado con la actividad turística, es la incorporación de la mujer como un agente económico relevante, transformándose en un pilar de la economía familiar. Esta característica viene a replicar las tendencias que se observan en otros espacios de la región (Garín, 2015). De esta manera, de los 32 emprendedores entrevistados, el 69\% corresponde al género femenino (22 personas).

\section{Gráfico 1: Distribución por grupos de edad y género emprendedores}

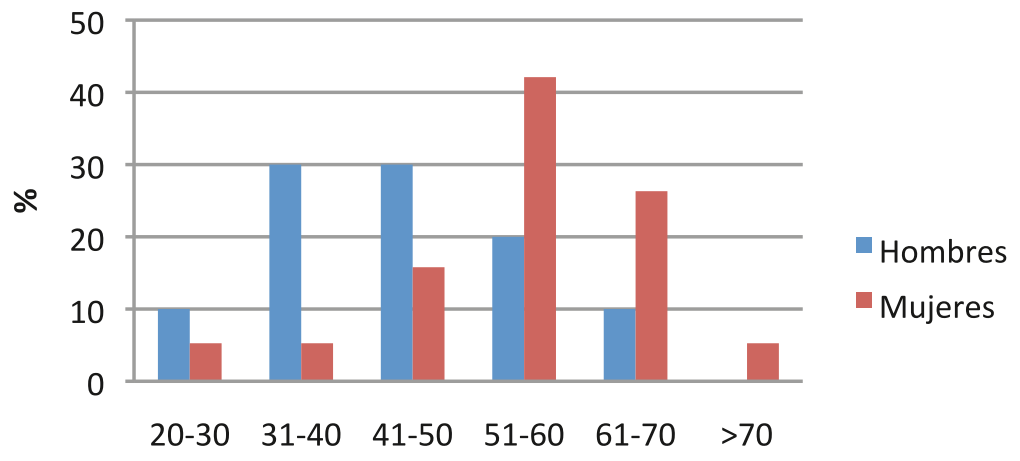

Grupos de edad

Fuente: Elaboración de los autores

El gráfico 1 muestra una importante diferencia en la distribución por edad y género. En el caso masculino, la concentración se encuentra entre 31 y 50 años de edad, en cambio, para las mujeres, 51 años y más. Esta situación, en especial en el caso de las mujeres, podría incidir en la sustentabilidad a través del tiempo de los emprendimientos. La distribución desigual se refuerza al considerar los promedios de edad, 46 y 56 años para hombres y mujeres respectivamente. De los entrevistados, la edad más baja corresponde a una mujer con estudios superiores de 24 años y la más alta, también a una mujer de 74 años con enseñanza media. Esta tendencia es similar a la encontrada en otras investigaciones en la cual se identifica que preferentemente adultos y adultos jóvenes son parte de la fuerza de trabajo en los espacios rurales (Garín, 2015) 


\section{Gráfico 2: Características del nivel de enseñanza de los emprendedores por género.}

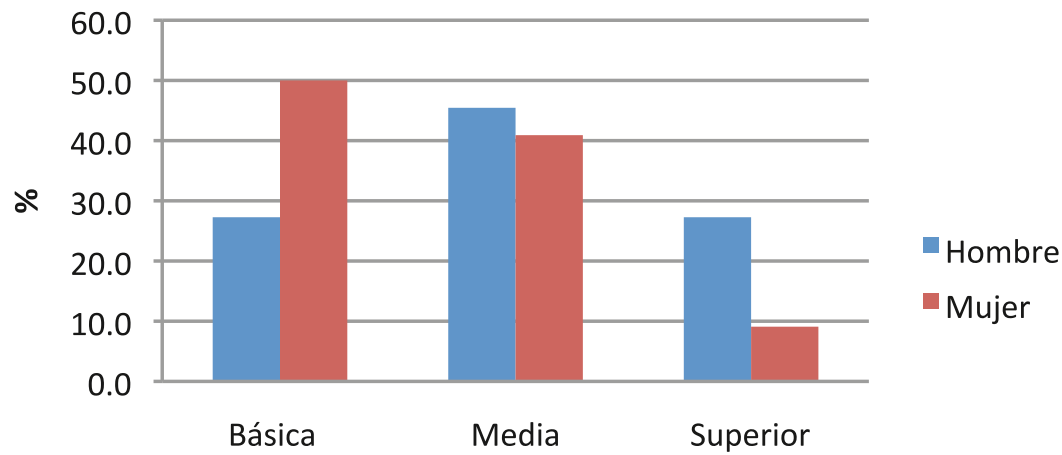

Tipò de enseñañza

Fuente: Elaboración de los autores

El gráfico 2, muestra las diferencias existentes en el nivel de enseñanza por género. En el caso de los hombres, se observa una concentración en la enseñanza media (45.5\%) y en las mujeres, en la educación básica (50\%). Otra diferencia se encuentra en la educación superior (población con más de 12 años de estudios), donde los hombres tienen una participación mayor (27\%) en relación a las mujeres (9\%).

No obstante que existe principalmente una población con estudios medios, el porcentaje que posee educación básica es importante, especialmente en las mujeres, aspectos que podrían dificultar la capacidad de innovación en sus emprendimientos.

\section{Gráfico 3: Principales actividades de los emprendedores turísticos.}

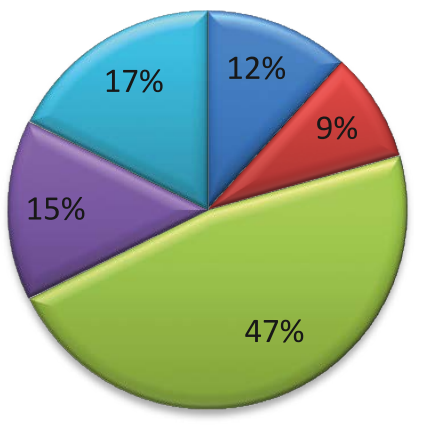

$\square$ Gastronomía

- Artesanía

$\square$ Alojamiento

$\square$ Productos tradicionales

Naturaleza

Fuente: Elaboración de los autores

En relación a las actividades que realizan los emprendedores, (gráfico 3), el alojamiento, ya sea en cabañas y/o camping, es la principal actividad y que en algunas oportunidades, se complementa con el agroturismo. Quienes entregan este servicio se localizan principalmente en las cercanías de los ríos que cruzan la comuna o en espacios que permiten contemplar el paisaje. La otra actividad en importancia, es la relacionada con la observación de la naturaleza. Estos emprendimientos funcionan principalmente en la temporada estival (53\%), un 38\% todo el año. El resto lo hace en invierno y en otoño- primavera. Quienes realizan su actividad durante todo el año, se dedican a la elaboración de 
productos artesanales y gastronomía, reciben delegaciones de turistas desde el extranjero, estudiantes universitarios provenientes desde Chile y también norteamericanas y en algunos casos, desde Europa.

\subsection{Fortalezas y debilidades desde la visión de los emprendedores turísticos}

Debido a la declinación de las actividades tradicionales que se ejercen en el espacio rural, la comunidad local ha vuelto su mirada a relevar las múltiples oportunidades que les ofrece el territorio comunal para desarrollar el turismo como una alternativa para mejorar su calidad de vida.

El TR es una actividad aún incipiente en la comuna y cuyos emprendimientos tienen diversos grados de desarrollo como resultado del conocimiento, experiencias, redes y capacidad económica. No obstante esta diversidad, hay elementos comunes que surgen de las entrevistas que dan cuenta del potencial que tiene el turismo para el desarrollo económico, no tan solo de los emprendedores, sino que también de la comuna.

Probablemente uno de los elementos más importantes y que puede ser considerado como una fortaleza para consolidar y hacer sustentable la actividad, es el empoderamiento e importancia que le asignan. En este sentido, los entrevistados (40\%) señalan que el turismo les permite rescatar y dar a conocer la cultura y hacer un uso sustentable de la naturaleza. A la vez, les genera un sustento económico que les ha permitido mejorar la calidad de vida y educar a sus hijos (40\%). Además, contribuye al desarrollo de las comunidades locales. Otro aspecto a destacar, es que es un ingreso complementario y que también les ha permitido una reestructuración productiva, utilizando racionalmente los recursos que poseen.

Su visión se traduce en las siguientes opiniones que reflejan lo señalado precedentemente.

"trabajaba en madera, la madera se terminó y ahora trabajo en turismo. La ganadería no da". "trabajamos en agricultura, esto ya murió hace tres años. El turismo da sustento a la familia"

"el turismo ha permitido mejorar nuestra calidad de vida. Contaba con cinco caballos, ahora tenemos 18 y construimos una ruca. Esto nos ha permitido utilizar y cuidar nuestro territorio y mostrando nuestra cultura"

"antes trabajaba en huerta. Ahora gastronomía mapuche y tenemos ruca y quincho. El retorno del dinero es más rápido, el dinero está en seguida. Solo con la ganancia del turismo hemos invertido"'.

En cuanto a las debilidades, la que más se repite, corresponde a la falta de formalización, principalmente sanitaria, donde el $45 \%$ no ha podido cumplir con este requisito. Algunos motivos que mencionan los emprendedores/as se relacionan con:

"existe una rigidez en la normativa, piden cosas que son adecuadas para la ciudad",

"el turista quiere conocer nuestra cultura y nos exigen cocina a gas para cocinar, cuando en el campo se usa cocina a leña",

"falta apoyo de la municipalidad para solicitar un cambio a medioambiente"

"Solo falta sanidad, ponen puras trabas, nos dijeron que como íbamos a mostrar un piso de tierra y todo tiene que ser lavable. Si tenemos una ruca"

La mirada de los emprendedores sobre la gestión municipal también es un factor que para ellos se transforma en una debilidad y que impide una adecuada integración entre la gestión pública y privada.

"El municipio debería más facilidades y que deberían haber técnicos que den capacitación. Y que pongan énfasis en el cuidado del ambiente. Debería buscar personas que asesoren y ayuden a mejorar los senderos para que el turista vea donde va el sendero"

"La municipalidad no ha apoyado. Estamos cansado de pedir",

"Municipalidad debería apoyar en entregar más publicidad, como tener algo mejor. Eso debería hacer el alcalde. Es una falencia".

"El municipio no tiene muchos recursos como otras comunas, por lo tanto, no es mucha la ayuda. La oficina de turismo no está bien armada, Es un tema que se debe arreglar a futuro".

Otro factor que valoran, pero que es considerado una debilidad, ya que ha sido difícil llevarlo a la práctica, es el trabajo comunitario.

"estamos organizados por teléfono y nos repartimos los turistas. Nos organizamos sin conflicto, cada uno hace algo específico. Cada hogar tiene su conocimiento y da su charla. Mientras más casas unidas, mejor para recibir turistas, para entregar un servicio bueno y parejo. Nos apoyamos en vez de competir. No vamos al supermercado. Lo compramos todo en la comunidad".

"en el sector tratamos de realizar turismo comunitario, pero no todos quieren hacerlo. Lo hemos logrado en algunos casos. Nosotros damos alojamiento, otros vecinos hacen las cabalgatas. Esto de común acuerdo. Creo que es la mejor manera de trabajar el turismo".

Se pudo percibir en las entrevistas que solo 2 personas tenían cierto conocimiento sobre el significado del turismo rural comunitario. Una de ellas, estudiante universitario y un joven con experiencia en otros países. Más que turismo comunitario, se percibe que es un trabajo asociativo e informal, ya que 
no forman parte de alguna estructura comunitaria funcional relacionada con el turismo. Se puede decir que es una asociatividad por proximidad geográfica, parentesco o intereses en común, como la preservación cultural y del paisaje.

\subsection{La localización del territorio como oportunidad estratégica}

Durante las entrevistas, los emprendedores mencionaron importantes oportunidades que les ofrece el territorio para realizar su actividad. Una de ellas es que la comuna es puerta de entrada a Chile desde Argentina. Esta percepción se origina a través de la observación de los flujos vehiculares. Lo anterior, se comprobó revisando las estadísticas de ingreso del paso internacional de Mamuil Malal.

\section{Gráfico 4: Entrada de pasajeros paso fronterizo Mamuil Malal. Comuna de Curarrehue}

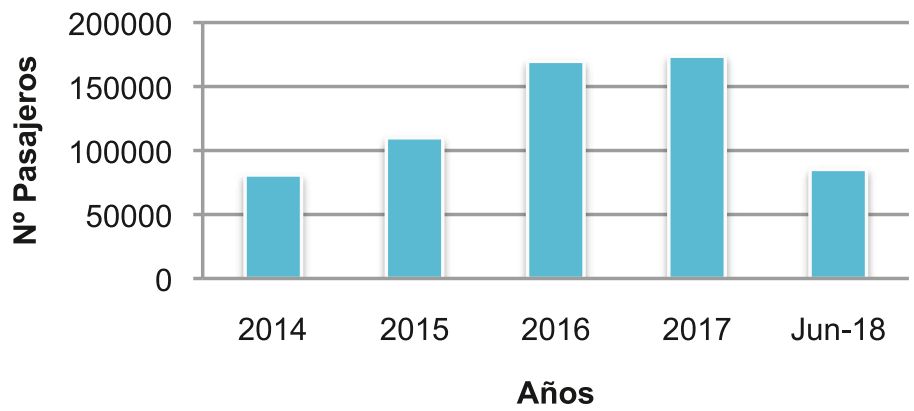

Fuente: Elaboración de los autores en base estadísticas SERNATUR 2018

En el gráfico 4, se observa el aumento en el ingreso de los turistas provenientes de la república Argentina y, por las tendencias que se han presdentado, es muy probable que continúen. Esta situacion revela la existencia de una oportunidad y a la vez un desafio para lograr que los visitantes se detengan y pernocten en la comuna.

\subsection{Las oportunidades asociadas al patrimonio cultural-histórico}

Se pusieron en valor las caracteristicas historicas, culturales y de naturaleza que posee el territorio. En este sentido, el territorio, por su conformación histórica, cuenta con una interesante presencia de comunidades mapuches y colonos, quienes a través del tiempo han ido construyendo un espacio que refleja la impronta de dos culturas y que se manifiesta, desde la actividad turística, en una variedad de elementos que se asocian principalmente al Turismo de Interese Espececiales (TIE). La presencia del pueblo mapuche se visibiliza a través de sus manifestaciones culturales tangibles e intangibles como es la articulación invernada- veranada, espacios que son utilizados para trasladar su ganado. Esta presencia permite interiorizarse sobre el conocimiento que poseen las comunidades y a la vez conocer espacios con alto contenido cultural como son eltunes, trayenco, winkules, raudales y pitrantus.

La figura 2 muestra la presencia de algunas actividades que desarrollan las comunidades mapuches y que permiten realizar un turismo experiencial compartiendo con las familias, aprender de gastronomía indígena, formas de vida y cosmovisión.

Desde el punto de vista histórico, por ser limítrofe con el territorio argentino, se encuentran pasos cordilleranos que permitían la articulación del ngulumapu con el puelmapu posibilitando un importante tráfico ganadero entre ambos espacios, los cuales poseían un sentido en la ritualidad mapuche constituyendo "puertas rituales" hacia las pampas. En estos trayectos también se identifican sitios como los chenques, similares a las cuevas y tipo de habitación semi-temporal que eran utilizadas como protección frente a las inclemencias del tiempo, lugar de preparación de alimentos, fabricación de herramientas de caza y faenamiento (Espinosa et. Al., 2013). 


\section{Figura 2: Nguillatuwe Chocol, gastronomía y ruka mapuche}
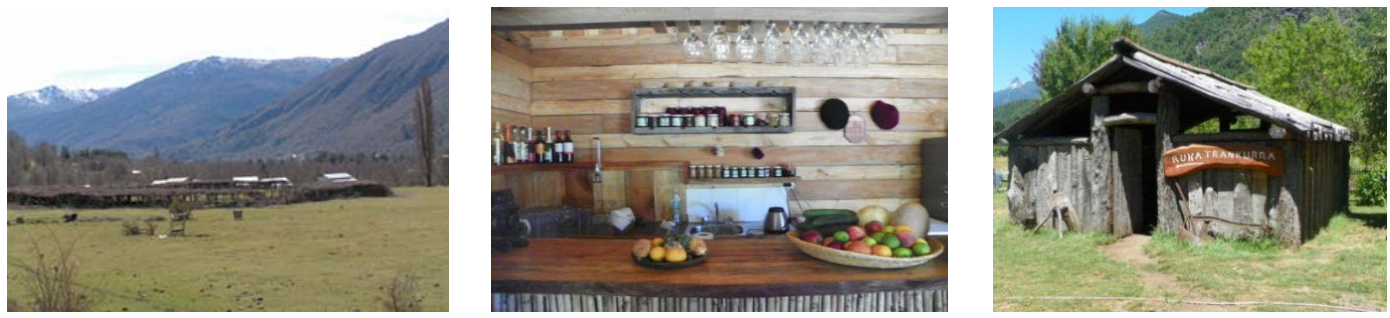

Fuente: Elaboración de los autores

\subsection{Las oportunidades asociadas a los recursos naturales para potenciar el turismo}

El espacio rural de Curarrehue posee importantes recursos asociados a la naturaleza de tipo geológicos, hidrológicos, geomorfológicos y de flora y fauna, lo cual otorgan a la comuna una posición privilegiada para realizar la actividad turística.

\section{Figura 2: Algunos Atractivos naturales (glacial volcán Sollipulli, Araucaria, laguna Hualalafquén)}
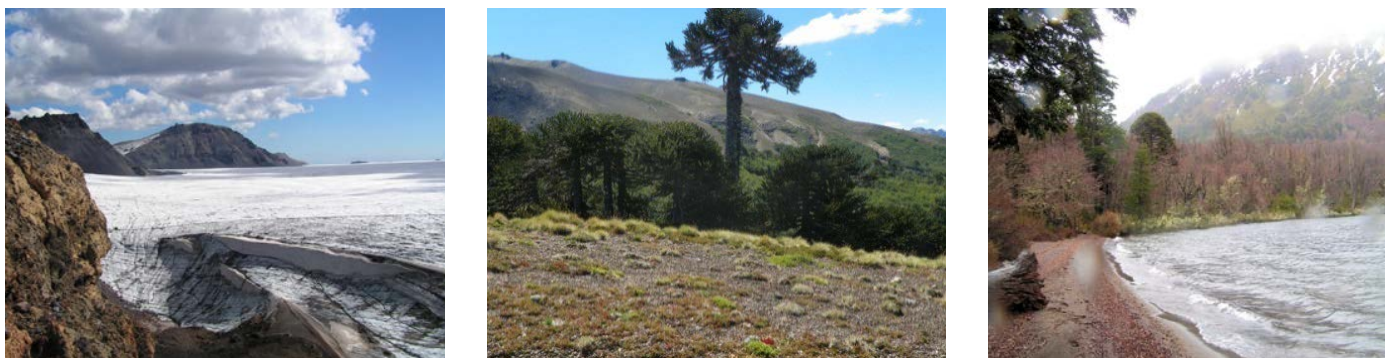

Fuente: Elaboración de los autores

La figura 2 muestra algunos de los atractivos de la comuna y que aún no se han desarrollado en plenitud. En la parte superior y a la izquierda, se observa el imponente glacial del volcán inactivo del Sollipulli. Este glaciar tiene la particularidad de tener el mayor volumen de hielo y altura al sur del Ecuador. En los alrededores del volcán se encuentra una cantera de obsidiana negra con abundante sustrato material que los mapuches en la antigüedad usaban para la elaboración de diferentes artefactos líticos como puntas de flecha y utensilios diversos. A la belleza intrínseca del lugar, se agrega la presencia de bosques nativos, donde sobresale la Araucaria, árbol de gran importancia ceremonial y económica para los mapuches de la pre-cordillera. Cabe destacar en este sector, la relación de la naturaleza con la cosmovisión del pueblo Mapuche, ya que al iniciar la ascensión al volcán, se ubica una Araucaria sagrada (figura 1 central), donde se realizan ceremonias rituales de agradecimiento o petición. Finalmente se observa la laguna Hualafquen, ubicada al interior de la reserva Nacional Villarrica, donde se encuentra una rica variedad de especies arbóreas nativas. Los recursos asociados a la naturaleza permiten efectuar diversas actividades relacionadas al turismo de intereses especiales (TIE), como Birdwatching, fotografía de flora, fauna y paisaje, pesca deportiva en todas sus modalidades (en laguna Hualalafquén y esteros efluentes), caminata (trekking), caminata a campo traviesa (hikking), remo (kayaking), canotaje, esquí (randonee), orienteering y cabalgatas (Espinosa et. Al., 2013).

Las oportunidades identificadas y su localización estratégica como puerta de entrada al territorio nacional y al compromiso que han adoptado los emprendedores para desarrollar la actividad, pueden permitir que este espacio periférico se transforme en un complemento al destino turístico de Pucón, uno de los más importantes de la región y sur de Chile, localizado a $30 \mathrm{~km}$ al poniente de la cabecera comunal.

\subsection{Las amenazas para el turismo rural}

La mayor amenaza que es percibida por los emprendedores, se asocia a la llegada de nuevas actividades productivas que no tienen relación ni beneficio para la comunidad local, como es el caso de las 
pisciculturas y centrales de paso, quienes poseen la propiedad de los derechos de agua, impidiendo a las comunidades aprovechar este recurso hídrico. Estas nuevas actividades han sido la causa de importantes conflictos, ya que se les considera que atentan fuertemente contra el turismo debido a la contaminación de los cursos de agua, la intervención del paisaje y la ocupación de sitios ceremoniales. Estos conflictos se han agravado debido al modo en que las nuevas empresas han llegado al territorio, generando la división de las comunidades. La percepción de los emprendedores se refleja en los siguientes comentarios.

"solo existe naturaleza viva y buenas aguas, por eso quieren invadir estos sectores"

"le da lo mismo, porque al final ganan los ricos. Al final los ríos están comprados"

"proyecto de agua es un proyecto invasivo que no aporta para el desarrollo local"

"soy defensora de la aguas y naturaleza y ya llegaron algunas invasiones que afectará a futuro. Evitar la hidroeléctricas y pisciculturas",

"Pisciculturas que no se sigan haciendo. Hace que el turismo se vaya. No queremos pisciculturas y centrales de paso".

"Una vecina había instalado un camping a orilla del rio. Venían turistas, pero cuando empezó a funcionar la piscicultura, las aguas se contaminaron y los turistas salian manchados. Al final tuvo que cerrar perdiendo la inversión".

Las empresas, a fin de validar su presencia en el territorio, han realizado prácticas que han dividido a las comunidades, incidiendo en la posibilidad de realizar un trabajo asociativo y comunitario. Lo anterior queda reflejado en las siguientes opiniones:

"llegó la empresa citando a reunión y nos hizo firmar un papel señalando que era la asistencia. Después ellos señalaron que nosotros habíamos firmado autorizando, puro engaño. Después, viendo la necesidad económica de los miembros de la comunidad, les ofrecieron dinero para que autorizaran la construcción de la central de paso. Algunos firmaron y eso ocasionó la división de la comunidad”.

"al igual que en otro sector, acá llegaron algunas autoridades que no eran del municipio acompañando a la empresa apoyando su instalación. Nosotros nos opusimos, ya que iba a generar un daño a nuestro recurso turístico que es el rio y paisaje. Algunos aceptaron el ofrecimiento y por el apoyo, los ayudaron a formar una cooperativa. Eso ocasionó un problema entre nosotros'”.

La llegada de actividades ajenas al territorio, da cuenta de la asimetría que existe en las relaciones de poder entre el gran capital y las comunidades locales. No obstante la fuerte oposición de la población, la legislación vigente no favorece las demandas de las comunidades locales, ya que en definitiva, tanto las centrales de paso como las pisciculturas han sido aprobadas por la institucionalidad pública.

Tabla 1: Resumen matriz FODA

\begin{tabular}{|c|c|c|c|}
\hline Fortalezas & Debilidades & Oportunidades & Amenazas \\
\hline Empoderamiento & Falta asociatividad & $\begin{array}{c}\text { Localización estratégica } \\
\text { de la comuna }\end{array}$ & $\begin{array}{c}\text { Piscicultura y Centrales } \\
\text { de paso }\end{array}$ \\
\hline $\begin{array}{c}\text { Importancia para } \\
\text { mejorar calidad de vida }\end{array}$ & $\begin{array}{c}\text { Desconocimiento } \\
\text { sobre turismo rural } \\
\text { comunitario }\end{array}$ & $\begin{array}{c}\text { Patrimonio cultural e } \\
\text { histórico }\end{array}$ & Propiedad del agua \\
\hline $\begin{array}{c}\text { Protección del paisaje y } \\
\text { patrimonio cultural }\end{array}$ & Relación con municipio & Naturaleza y paisaje & \\
\hline & Informalidad sanitaria & & \\
\hline
\end{tabular}

Fuente: Elaboración de los autores

Los resultados obtenidos son concordantes con la literatura que apunta a que el TR es una actividad que contribuye al desarrollo y mejoramiento de la calidad de vida de los emprendedores (Pérez de Murzi, 2007; Flores e Silva, Cunha ,Angelo y Christoffoli, 2016; Rummenhoeller, 2012) como también que existen dificultades para trabajar en forma asociativa (Gascón, 2016).

\section{Conclusiones}

De manera similar a otros espacios rurales, el TR en Curarrehue, ha surgido como una nueva alternativa de desarrollo que permite incrementar los ingresos y que desde la perspectiva de la NR, tal 
como lo plantea Palafox (2016), es una actividad complementaria a las tradicionales, permite preservar la naturaleza, dar a conocer su cultura, mejorar su calidad de vida y contribuir al desarrollo de las comunidades y además, tal como se ha identificado en esta investigación, es parte de una reestructuración productiva (Pérez, 2010; Galmarini, 2016).

No obstante la existencia por parte de los emprendedores de fortalezas y oportunidades, se presentan ciertas debilidades y amenazas que pueden incidir en la sustentabilidad de los emprendimientos, como ser la falta de asociatividad, la cual impide el encadenamiento entre sus iniciativas, la percepción de ausencia de la autoridad local y escaso involucramiento con los emprendedores. A la vez, la mayor amenaza que es percibida, es la competencia por la utilización de recursos naturales escasos debido a la llegada de nuevas actividades como las pisciculturas y centrales de paso, ambas asociadas a capitales externos al territorio, lo cual ha generado diversos conflictos, visibilizando una fuerte asimetría en las relaciones de poder ampliamente favorables al capital globalizado, situación que no es ajena a otros espacios rurales, donde este capital entra a competir con las actividades que tradicionalmente han realizado las comunidades locales (Jerez, 2015; Sañudo, Quiñones, Copete et al, 2016)

Teóricamente, la perspectiva de la construcción social del turismo y compartiendo lo indicado por Kieffer (2018), es un enfoque adecuado que permite conocer como el territorio, a partir de la acción de los actores sociales y locales han ido desarrollando la actividad turística como producto de su historia, conocimiento y saberes patrimoniales.

Teniendo en cuenta que esta investigación es un enfoque parcial sobre los actores del turismo, queda el desafío de conocer la relación existente con los turistas y como la población local percibe si la actividad contribuye efectivamente al desarrollo .del territorio.

Agradecimientos: Los autores agradecen a la Dirección de Investigación de la Universidad de la Universidad de La Frontera, el apoyo entregado para realizar la investigación a través del proyecto DIUFRO: DI14-0048 "Los impactos socioespaciales de la globalización en la comuna de Curarrehue. 1992- 2013".

\section{Bibliografía}

Avram, D.;Balan, A. 2015. "Innovative methods in the development of rural tourism in Romania". Junior Scientific Researcher. 1 (1): 33-41.

Banco Mundial; Fao, 2003. La nueva ruralidad en Europa y su interés para América Latina. Consultado 2 de marzo 2016. http://www.fao.org/docs/eims/upload/249910/Y4524sLA.pdf.

Bardón, E. 1990.” Consideraciones sobre el turismo rural en España y medidas de desarrollo". Estudios Turísticos, (108): 61-82

Barrera, E. 2006. “Turismo rural: nueva ruralidad y empleo rural no agrícola”. CINTERFOR/OIT.185 pág. Consultado 10 de abril 2018 en: http://www.agro.uba.ar/sites/default/files/turismo/publicaciones/ barrera/TR_Y_EMPLEO_OIT_2006.pdf.

Barke, R. 2004. "Rural Tourism in Spain". International Journal of Tourism Research. 6. (3): 137-149.

Berger, P. y Luckman, T. 1968. "La construcción social de la realidad. Amorrortu, Buenos Aires

Bianchin, A. y Alves, B. 2017. "Cultura e mercadoria: perspectivas do turismo comunitário na América Latina”. Revista Latino-Americana de Estudos em Cultura e Sociedade. 3:1-21. Consultado 20 junio 2018 en: http://periodicos.claec.org/index.php/relacult/article/view/828

Briedenhann, J.; Wickens, E. 2004. "Rural tourism - meeting the challenges of the new South Africa". International Journal of Tourism Research, 6(3): 189-203.

Cañada, E. y Fandiño, M. 2009. Experiencias de turismo comunitario en Nicaragua. Aportes a la economía campesina. Colección Buenas Prácticas. EDISA, Nicaragua.

Cardona, J.; Sierra, A. 2013. "Peculiaridades del turismo rural de Baleares". Gestión Turística. (20):9-38.

Cohen, E. 2005. "Principales tendencias en el turismo contemporáneo". Política y Sociedad. 42(1):11-24.

Corbetta, P. 2007. "Metodología y técnicas de investigación social”. Ed. Mc. Graw Hill.

Costa, A.M. y Barretto, M. 2007. "Los cambios socioculturales y el turismo rural: el caso de una posada familiar". Pasos. Revista de Turismo y Patrimonio Cultural. 5 (1): 45-52

Delgado, J.M. 2015. "Impactos del turismo desde la perspectiva de la sociología del turismo". XVIII congreso AECIT. Consultado el 3 de julio 2018 en: https://www.aecit.org/estudio-del-impacto-ambiental-del-turismo-rural/congress-papers/47/. 
De Souza, L. 2012. "Relación ciudad-campo y turismo rural. Ensayos teórico-metodológicos. Estudios y Perspectivas en Turismo, 21(1):1-19.

Espinosa, A,; Tereucan J. 2013. "Diseño de circuitos de Turismo de Intereses Especiales basado en recursos naturales, culturales e históricos". En, Turismo de Interese Especiales. Experiencias desde la Investigación Científica. Centro de Investigaciones Territoriales, Universidad de La Frontera, Temuco.

Flores, D.; Barroso, M. 2012. "El turismo como estrategia de desarrollo rural sostenible: los parques naturales andaluces". Revista de Estudios Empresariales. Segunda época. (1): 59 - 83.

Flores, Y; Cunha, F.; \& Christofolli, A. 2016. "Turismo rural comunitario. Gestión familiar y estrategias de consolidación en el Estado de Santa Catarina, Brasil”. Estudios y Perspectivas en Turismo. 25: $576-596$.

Fuller, N. 2011. "Reflexiones sobre el turismo rural como vía de desarrollo. El caso de la comunidad de Antioquía, Perú". Estudios y Perspectivas en Turismo. 20: 929-942.

García, B. 2005. "Características diferenciales del producto turismo rural". Cuadernos de Turismo, (15):113-133.

Garín, A. 2015. "Turismo Rural en la comuna de Villarrica, Chile. Institucionalidad y emprendedores rurales". Estudios y Perspectivas en Turismo. 24(1): 21-39.

Garín, A.; Albers, C.; Ortega, E. 2011."Las expresiones de la ruralidad en la región de La Araucanía, Chile, 1997-2007". Revista Estudios Sociales de Hermosillo. 19 (38): 69-89

Garcia, J.L. 1996. "El turismo rural como factor diversificador de rentas en la tradicional economía agraria". Estudios Turísticos, (132): 47-61.

Galmarini, M. 2016. "Turismo rural y desarrollo económico local; el caso del partido de Lobos, Provincia de Buenos Aires". Revista de Ciencias Sociales segunda época. (29):35-51.

Gascón, J. 2016. "Limitaciones del Turismo Rural Comunitario como instrumento de Cooperación Internacional. El concepto de "vocación social del territorio". Actas I Congreso COODTUR. Tarragona: Publicaciones URV. 2010. Consultado el 20 de mayo 2018 en: www.coodtur.org/wp-content/ uploads/2016/04/Mesa-3.-I-Congreso.pdf.

Gómez, S. 2002. "Nueva Ruralidad. (Fundamentos teóricos y necesidad de avances empíricos). Seminario internacional "el mundo rural: transformaciones y perspectivas a la luz de la nueva ruralidad". Consultado el 15 de julio 2018 en: http://www.javeriana.edu.co/ier/recursos_user/documentos/ revista50/167_168.pdf

González, A. 2011. Construcción social del turismo Una perspectiva teórica de sociología constructivista para el estudio del turismo. Editorial Académica Española. Alemania.

González, M y Palafox, A. 2014. "Sociología del turismo en español. Revisión exploratoria de artículos publicados en revistas iberoamericanas 2003 - 2013. Estudios y Perspectivas en Turismo. 23: 805-819

Grajales, S.; Concheiro, L. 2009."Nueva ruralidad y desarrollo territorial. Una perspectiva desde los sujetos sociales". Veredas. Revista del Pensamiento Sociológico. (18):145-167

Hall, M. (S/F). "Sustainable rural areas tourism development and issue. Consultado el 15 de mayo 2017 en: http://www.edsconference.com/content/docs/papers/Hall,

Hwang, J. y Lee, S. 2015. "The effect of the rural tourism policy on non-farm income in South Korea". Tourism Management, (46): 501-513.

INDAP 2012. "Resolución que aprueba Programa de Turismo Rura"l. Consultado 15 de abril 2017 en: http:// www.indap.gob.cl/sites/default/files/documentos_relacionados/resolucion_norma-turismorural-2012.

Instituto Nacional de Estadísticas 2003.” Censo de Población y Vivienda 2002”.en: Consultado el 15 julio 2018 en: www.ine.cl

Instituto Nacional de Estadísticas 2017.” Censo de Población y Vivienda 2017”. Consultado el 15 julio 2018: www.ine.cl

Jerez, B. 2015. "Conflictos ecoterritoriales transfronterizos y megaproyectos extractivistas en cuencas compartidas de la Patagonia. Revista Nuestra América. 3(5): 56-73

Kastenholz, E.; Carneiro, M.; Peixeira Marques, C.; Lima, J. 2012. "Understanding and managing the rural tourism experience. The case of a historical village in Portugal". Tourism Management Perspectives. (4): 207-214.

Kay, C. 2007. "Algunas reflexiones sobre los estudios rurales en América Latina”. Iconos. Revista de Ciencias Sociales. (29): 31-50

Kay, C. 2009. "Estudios rurales en América Latina en el periodo de globalización neoliberal: ¿una nueva ruralidad? Revista Mexicana de Sociología, 71 (4): 607-645

Kieffer, M. 2018. "Turismo rural comunitario y organización colectiva: un enfoque comparativo en México" Pasos, Revista de Turismo, y Patrimonio Cultural. 16 (2): 429-441. 
Komppula, R. 2014. "The role of individual entrepreneurs in the development of competitiveness for a rural tourism destination. A case study". Tourism Management (40): 361-371.

Lane, B. 1994. "Tourism strategies and rural development". Organisation for Economic Cooperation and Development.

Llambi, L. y Pérez, E. 2007. "Nuevas ruralidades y viejos campesinismos. Agenda para una nueva sociología rural latinoamericana. Cuadernos de Desarrollo Rural, (59):37-61

Marujo, M. y Cravidão, F. 2012. "Turismo e lugares: uma visão geográfica”. PASOS - Revista de Turismo y Patrimonio Cultural 10(3): 281-288

Milano, C. y Gascón, J. 2017. "Turismo y sociedad rural, o el extraño caso del doctor Jekyll y Mr. Hyde". Pasos. Revista de Turismo y Patrimonio Cultural. Colección Pasos, Edita ${ }^{\circ} 18$.

Millán, M.G.; López-Guzmán; Agudo, E. 2006. "El turismo rural como agente económico: desarrollo y distribución de la renta en la zona de Priego de Córdoba”. Revista de Economía Pública, Social y Cooperativa (55):167-192

Monterroso, N. y Zizumbo, L. 2009. "La reconfiguración neoliberal de los ámbitos rurales a partir del turismo: ¿avance o retroceso?". Convergencia 16(50): 133-164. Consultado el 6 de junio 2017 en: http:// www.scielo.org. $\mathrm{mx} / \mathrm{scielo}$.php?pid=S1405-14352009000200006\&script=sc.

Neumeier, S. y Pollermann, K. 2014. "Rural tourism as promoter of rural development. Prospects and limitations: case study findings from a pilot project promoting village tourism". European Countryside. (4): $270-296$.

Nogar, A. 2007. "La multifuncionalidad territorial como escenario de la nueva ruralidad". Pampa (3):27-41.

Pastor, J.; Jurado, C. y Soler, A. 2011. "Desarrollo rural a través del turismo comunitario. Análisis del valle y cañón de Colca". Gestión Turística (15):1-20

Municipalidad de Curarrehue 2010. "Plan de Desarrollo Comunal 2010-2016".

Pérez de Murzi, T. 2007. "La actividad turística como opción de desarrollo para las áreas rurales del estado Táchira, Venezuela. El caso de San Vicente de la Revancha. Revista Scripta Nova. Consultado el 23 de mayo 2018 en: http://revistes.ub.edu/index.php/ScriptaNova/article/view/1383.

Pérez. S. 2010. "El valor estratégico del turismo rural como alternativa sostenible de desarrollo territorial rural". Agronomía Colombiana. 28 (3): 507-513

Ramírez, C. 2014. "Visión crítica sobre los enfoques de la Nueva Ruralidad y el desarrollo territorial rural en América Latina”. Agronomía Colombiana 32(1):122-129.

Randelli, F.; Romei, P. y Tortora, M. 2014. "An evolutionary approach to the study of rural tourism: The case of Tuscany". Land Use Policy. (38):276-281.

Rummenhoeller, K. 2016. "De la expectativa a la desilusión: turismo comunitario en la región del manu (Perú)".Pasos. Revista de Turismo y Patrimonio Cultural. Colección Pasos, Edita nº6.

Sariego, J.; Pons, I., Serrano, M. y Vera. F. 2016. "El papel del turismo en un entorno nuevo y cambiante: el turismo comunitario como práctica de desarrollo de las áreas rurales del Perú”. Consultado el 23 de junio 2018 en: www.aecit.org/uploads/public/congresos/16/Comunicaciones/\%les.

Sampaio, C. y Coroliano, L. 2009. "Dialogando com experiências vivenciadas em Marraquech e América Latina para compreensão do turismo comunitário e solidário. Revista Brasileira de Pesquisa em Turismo. 3 (1): 4-24

Sánchez, A. 2014. "El turismo rural y su incidencia en el desarrollo socioeconómico de un territorio: El caso de la comarca de La Sidra”. Tesis de grado, para optar al Master en Dirección y Planificación del Turismo. Universidad de Oviedo, España. Consultado 22 de junio 2017 en: http://digibuo.uniovi. es/dspace/bitstream/10651/27986/1/TFM_Adriana\%20Sanchez\%20Rivera.pdf.

Sánchez, A. 2016. "Sociología rural y nueva ruralidad sur-sur". Espacio Abierto, 25 (3): 49-63

Sañudo, M; Quiñones, A.; Copete, J.; Díaz, J.; Vargas, N. y Cáceres, A. 2016. "Extractivismo, conflictos y defensa del territorio: el caso del corregimiento de La Toma Cauca, Colombia". Desafíos, 28(2): 367-409

Servicio Nacional de Turismo. 2018. "Estadísticas de pasajeros por pasos fronterizos". Consultado el 7 de junio 2018 en: www.sernatur.cl

Sharpley, R. 2002. "Rural tourism and the challenge of tourism diversification: the case of Cyprus". Tourist Management. 23 (3):233-244.

Snieška, V.; Barkauskienè, K. y Barkauskas, V. 2014. "The Impact of Economic Factors on the Development of Rural Tourism: Lithuanian Case". Procedia- Social and Behavioral Sciences. (156): 280-285. Consultado el de mayo 2018 en: http://doi.org/10.1016/j.sbspro.2014.11.1892014.

Tejera, J. 2010. "Turismo comunitario: soluciones para una adecuada comunicación y comercialización”. Consultado el 15 de julio 2018 en: http://www.ecotumismo.org/turismo-responsable/turismo-comunitario 
Varisco, C. 2016. "Turismo Rural: Propuesta Metodológica para un Enfoque Sistémico". PASOS - Revista de Turismo y Patrimonio Cultural. 14(1): 153-167

Vásquez, C. y Martin, F. 2011. "Problemas de sostenibilidad del turismo rural en España". Anales de Geografía. 31(1): 171-194

Zou, T.; Huang, S. y Ding, P. 2014. "Toward A Community-driven Development Model of Rural Tourism: the Chinese Experience". International Journal of Tourism Research. (16): 261-271. 